\title{
Vascular Closure Devices after Endovascular Procedures in Swine: A Reliable Method?
}

\author{
P. Isfort, ${ }^{1}$ T. Tanaka, ${ }^{2}$ T. Penzkofer, ${ }^{1}$ P. Bruners, ${ }^{1}$ R. Tolba, ${ }^{3}$ \\ C. K. Kuhl, ${ }^{1}$ and A. H. Mahnken ${ }^{1,4}$ \\ ${ }^{1}$ Department of Diagnostic and Interventional Radiology, RWTH Aachen University Hospital, Pauwelsstrasse 30, \\ 52074 Aachen, Germany \\ ${ }^{2}$ Department of Radiology, Nara Medical University, 840 Shijo-cho, Kashihra 634-8522, Japan \\ ${ }^{3}$ Institute of Animal Research, RWTH Aachen University Hospital, Pauwelsstrasse 30, 52074 Aachen, Germany \\ ${ }^{4}$ Department of Diagnostic and Interventional Radiology, Philipps-University, Baldingerstrasse, 35043 Marburg, Germany
}

Correspondence should be addressed to P. Isfort; isfort@ukaachen.de

Received 3 December 2013; Accepted 21 January 2014; Published 11 March 2014

Academic Editors: W. S. Aronow and B. F. Uretsky

Copyright (C) 2014 P. Isfort et al. This is an open access article distributed under the Creative Commons Attribution License, which permits unrestricted use, distribution, and reproduction in any medium, provided the original work is properly cited.

\begin{abstract}
Purpose. To investigate the safety and feasibility of the use of a vascular closure device (VCD) after endovascular procedures in swine. Material and Methods. In a study on endovascular therapy, VCD (StarClose, Abbott Vascular, Il, USA) was used in 20 female swines to achieve immediate hemostasis after percutaneous right femoral artery (FA) access. 10 animals were sacrificed immediately after the study and 10 animals were sacrificed 28 days after the initial study. To ensure complete hemostasis and patency of the femoral artery, a CT-angiography of the puncture site was performed on day 1 (acute and chronic group) and day 28 (chronic group). After the sacrifice, the femoral artery was explanted and examined macroscopically for signs of VCD dysfunction. Results. Technical success rate was $100 \%$ with immediate hemostasis being achieved in all animals. No animals showed evidence of hematoma. During explantation, only small traces of coagulated blood were found in the acute group, while there were no signs of hematoma in the chronic group. CT-angiography immediately after VCD application as well as before sacrifice (chronic group) showed patency of the FA in all cases. Conclusion. The use of VCD to achieve hemostasis after endovascular studies in swine is feasible and safe.
\end{abstract}

\section{Introduction}

Vascular closure devices are increasingly used after various types of endovascular procedures including coronary, cerebrovascular, and peripheral vascular interventions as an alternative to manual compression to achieve hemostasis after cannulation of the right femoral artery (FA). Manual compression of the puncture site is associated with prolonged bed rest and a local complication rate (hematoma, bleeding, pseudoaneurysm, and arteriovenous fistula) of up to $9 \%$ [1-3]. In contrast, the systematic use of VCD can lower the local complication rate to as low as $0.53 \%$ [4]. Nevertheless, the superiority of VCD compared to manual compression with regards to bleeding complications has not been demonstrated in a randomized-controlled trial comparing the methods directly. Minimizing access site complications, patient immobilization, shortening patients' hospital stay, and thereby minimizing the costs of these interventions are the key towards the success of percutaneous endovascular procedures.
In endovascular studies, involving FA cannulation of swine manual compression and prolonged rest is not an option since the animals will move as soon as they awake from anesthesia. Moreover, due to the different anatomical situation in swine with the FA located deeper than in humans, sufficient compression of the punctured vessel against the femoral head is not possible. In this scenario, the use of VCD is indispensable. To date, no information about the safety and efficacy of VCD in swine is available.

The aim of this study is to examine the safety and feasibility of the use of VCD (StarClose) to achieve hemostasis after puncture of the FA.

\section{Material and Methods}

2.1. Study Design. In the context of an animal endovascular study, the puncture site in the right FA was closed by means of VCD in $n=20$ female swine. $N=10$ animals were sacrificed after the immediate CT-control (acute group) and $n=10$ 


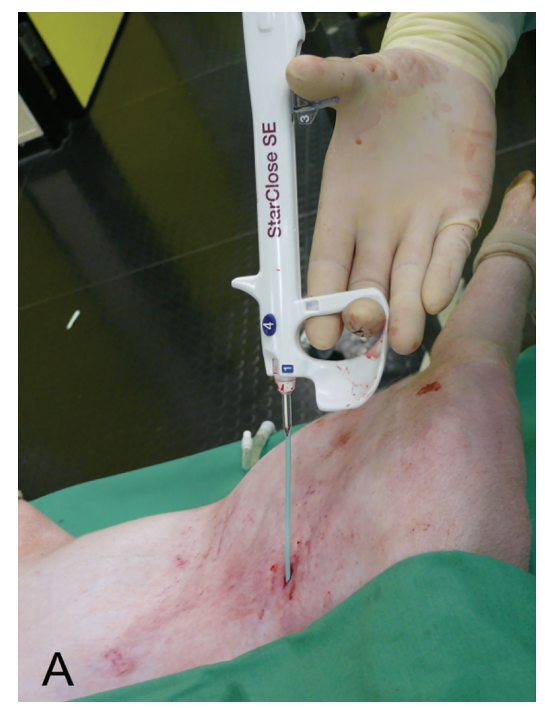

(a)

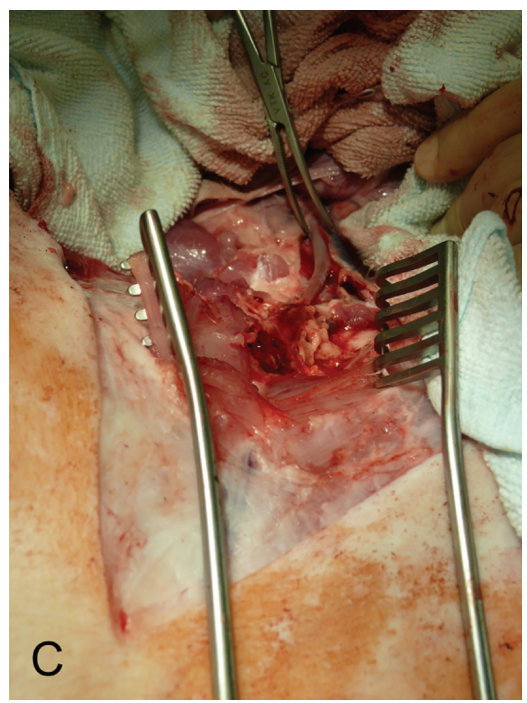

(c)

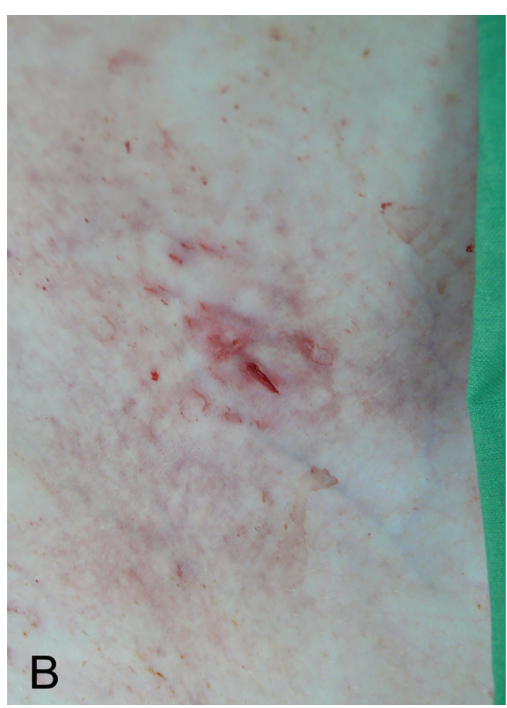

(b)

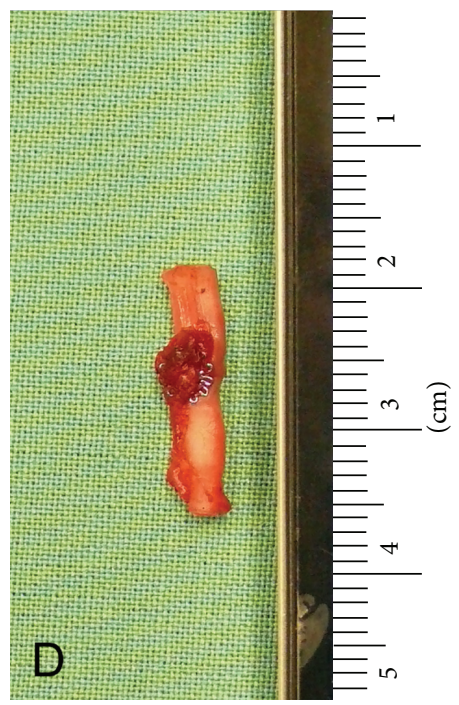

(d)

Figure 1: (a) Application of the VCD in pigs right FA. (b) No bleeding after application of the VCD. (c) Only subtle subcutaneous bruising and no significant hematoma visible in dissection of the right groin. (d) Right FA specimen with metallic clip affixed to the vessel wall.

animals were sacrificed 28 days after the procedure and after a repeated CT-control (chronic group).

After animals were sacrificed, the punctured groin was carefully dissected in order to evaluate local complications like hematoma or laceration of the FA. In one exemplary case, the right FA was excised in the region of the puncture site for photographic documentation.

2.2. Animal Experiments. All experiments were performed in accordance with the German legislation governing animal studies. Official permission was granted from the governmental animal care office (Landesamt für Natur, Umwelt und Verbraucherschutz Nordrhein-Westfalen, Recklinghausen, Germany). Female German landrace pigs from a disease-free barrier breeding facility were housed in ventilated rooms and allowed to acclimatize to their surroundings for a minimum of 5 days before surgery. The animals, weighing around $60 \mathrm{~kg}$, were fasted $12 \mathrm{hrs}$ prior to the experiments. For premedication, the animals received an intramuscular injection of $4 \mathrm{mg} \mathrm{kg}^{-1}$ azaperone (Stresnil, Janssen, Germany). Anesthesia was induced by intravenous injection of $3 \mathrm{mg} \mathrm{kg}^{-1}$ propofol followed by oral intubation. The animals were ventilated with $40 \%$ oxygen at $20-26 \mathrm{bpm}$ and a tidal volume of $10 \mathrm{~mL} \mathrm{~kg}^{-1}$ to keep the end tidal partial carbon dioxide tension (pCO2) between 36 and $42 \mathrm{mmHg}$. Anesthesia was maintained with isoflurane at a concentration of $1 \%-1.5 \%$ (Forane, Abbott, Germany) and fentanyl (fentanyl, Janssen, Germany) at a concentration of $3-4 \mathrm{mg} \mathrm{kg}^{-1}$. To compensate for basic fluid requirements volume, animals received Ringer's lactate (RL) solution at a rate of $4 \mathrm{~mL} \mathrm{~kg}^{-1}$; after laparotomy, the constant infusion rate was set to $8 \mathrm{ml} \mathrm{kg}^{-1}$ and not changed until infliction of trauma. 


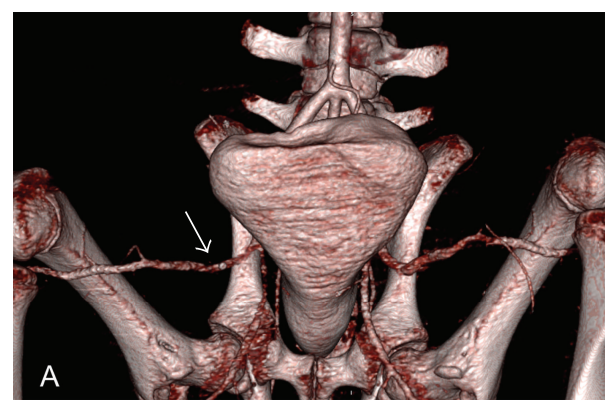

(a)

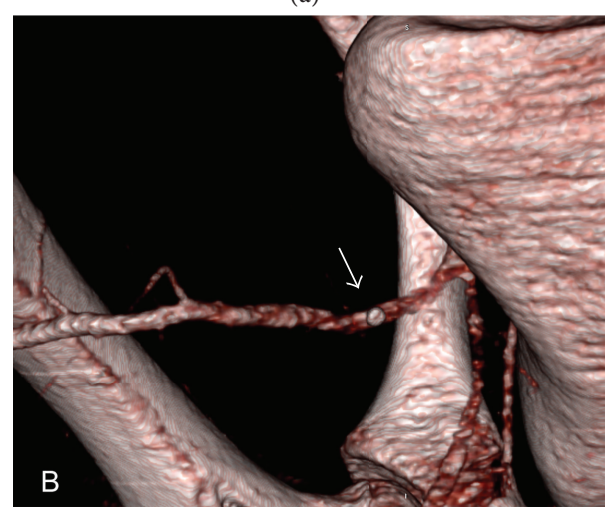

(b)

FIGURE 2: Three-dimensional visualization of a CT dataset in arterial contrast phase using volume rendering technique (VRT). (a) VRT after application of the VCD and (b) close-up of right FA with VCD clip in situ. Note unimpeded patency of the right femoral artery after VCD application. Note markedly different anatomy when compared to human vasculature.

2.3. Vascular Closure Device. An FDA-approved VCD (StarClose, Abbott Vascular; Abbott Laboratories, Abbott Park, Il, USA) using a clip-mediated sealing mechanism was used in this study. Therefore, the vascular sheath $(5 \mathrm{~F})$ in the FA has to be exchanged over a stiff guide wire for the system specific sheath. Thereafter, the tip of the system containing the sealing clip is introduced into the sheath until the system clicks into place. After that, the sheath is split and the sealing clip closes the puncture site. No local compression of the puncture site is performed.

2.4. Imaging Follow-Up. After applying the VCD contrastenhanced Angio-CTs after administration of $123 \mathrm{~mL}$ iopromide (Ultravist 300, Bayer Healthcare, Berlin, Germany) using a 64-slice dual-source CT (Siemens SOMATOM Definition, Siemens Healthcare Sector, Erlangen, Germany). Imaging follow-up was performed immediately after the intervention (acute and chronic group) as well as 28 days after the intervention (chronic group). Exemplary CT visualization using volume rendering technique (VRT) was performed using OsiriX (Version 5.6, 64 bit, http://www.osirixviewer.com/).

\section{Results}

In the control-CTs after the intervention, technical success rate was $100 \%$ with no signs of occlusion or extravasation and preserved blood flow in the FA in all animals. Clinical success rate was also $100 \%$ with preserved perfusion of the FA in the control-CTs 28 days after vascular closure.

After the animals were sacrificed, no signs of significant complications like significant hematoma surrounding the puncture site or laceration of the FA were observed. The metallic clip closing the puncture site was visibly affixed to the artery wall in all cases. In the acute group, small amounts of blood were visible in the region of the puncture site, which can be considered as normal leakage during the intervention. See also Figure 1 for VCD application and resected artery specimen.

\section{Discussion}

Vascular closure devices (VCD) aim at shortening of haemostasis time and reduction of peripheral vessel complications after arterial puncture. Today, a number of different VCDs are available which can be subclassified into four groups: (1) collagen-based systems (e.g., Angio-Seal, St. Jude Medical, Minnetonka, MN, USA), (2) surface pads with haemostatic substances (e.g., Chito-Seal, Abbott Vascular Devices, CA, USA) as an assisted manual compression, (3) suture-mediated or rather clip closure systems (e.g., StarClose, Abbott Vascular Devices, CA, USA), and (4) multiplecomponent systems (e.g., DUETT, Vascular Solutions, Minneapolis, MN, USA).

All the aforementioned systems have significant benefits, such as reduction of time to haemostasis and early ambulation of patients and therefore less rates of secondary complications like deep vein thrombosis and pulmonary embolism, an increased patient comfort, and earlier discharge for some patients [4-14]. Nowadays, the use of VCDs causes no increase in access site complications compared to conventional manual compression [2, 3]. With VCD optimization and increasing experience of the physicians, a trend towards reduced local complications through the use of VCDs in comparison to manual compression can be observed $[5,7,9,15]$. To date, no data on hemostasis after FA puncture using VCD in swine is available. Since the local anatomy of the vasculature in the pigs groin is different from the anatomy in humans (see also Figure 2), evaluation of the application and success of hemostasis are mandatory before routinely using VCD in swine. Moreover, due to the comparably deep localization of the FA in swine, a controlled compression of the FA against the femoral head is not possible.

In conclusion, hemostasis was successfully achieved in all animals while patency of the punctured FA was preserved. Thereby, no additional local compression was necessary.

\section{Limitations}

The presented study evaluates the safety of one particular VCD-the StarClose-Device. So, results cannot be transferred directly to other systems. Moreover, the study evaluated a rather small sample size when compared to studies evaluating VCD in humans, but, in our opinion, the sample 
size is sufficient for routine application of the system in animal trials.

\section{Conflict of Interests}

The authors declare that they have no conflict of interests regarding the publication of this paper.

\section{References}

[1] T. K. Nasser, E. R. Mohler III, R. L. Wilensky, and D. R. Hathaway, "Peripheral vascular complications following coronary interventional procedures," Clinical Cardiology, vol. 18, no. 11, pp. 609-614, 1995.

[2] N. A. Omoigui, R. M. Califf, K. Pieper et al., "Peripheral vascular complications in the Coronary Angioplasty Versus Excisional Atherectomy Trial (CAVEAT-I)," Journal of the American College of Cardiology, vol. 26, no. 4, pp. 922-930, 1995.

[3] R. Waksman, S. B. King III, J. S. Douglas et al., "Predictors of groin complications after balloon and new-device coronary intervention," The American Journal of Cardiology, vol. 75, no. 14, pp. 886-889, 1995.

[4] H. Eggebrecht, M. Haude, U. Woertgen et al., "Systematic use of a collagen-based vascular closure device immediately after cardiac catheterization procedures in 1,317 consecutive patients," Catheterization and Cardiovascular Interventions, vol. 57, no. 4, pp. 486-495, 2002.

[5] B. Chevalier, B. Lancelin, R. Koning et al., "Effect of a closure device on complication rates in high-local-risk patients: results of a randomized multicenter trial," Catheterization and Cardiovascular Interventions, vol. 58, no. 3, pp. 285-291, 2003.

[6] E. K. Hoffer and R. D. Bloch, "Percutaneous arterial closure devices," Journal of Vascular and Interventional Radiology, vol. 14, no. 7, pp. 865-885, 2003.

[7] W. G. Kussmaul III, M. Buchbinder, P. L. Whitlow et al., "Rapid arterial hemostasis and decreased access site complications after cardiac catheterization and angioplasty: results of a randomized trial of a novel hemostatic device," Journal of the American College of Cardiology, vol. 25, no. 7, pp. 1685-1692, 1995.

[8] S. Le Sage, M. McGee, and J. D. Emed, "Knowledge of Venous Thromboembolism (VTE) prevention among hospitalized patients," Journal of Vascular Nursing, vol. 26, no. 4, pp. 109-117, 2008.

[9] V. Legrand, P. Doneux, C. Martinez, O. Gach, and M. Bellekens, "Femoral access management: comparison between two different vascular closure devices after percutaneous coronary intervention," Acta Cardiologica, vol. 60, no. 5, pp. 482-488, 2005.

[10] W. Mlekusch, P. Dick, M. Haumer, S. Sabeti, E. Minar, and M. Schillinger, "Arterial puncture site management after percutaneous transluminal procedures using a hemostatic wound dressing (Clo-Sur P.A.D.) versus conventional manual compression: a randomized controlled trial," Journal of Endovascular Therapy, vol. 13, no. 1, pp. 23-31, 2006.

[11] T. A. Sanborn, H. H. Gibbs, J. A. Brinker, W. D. Knopf, E. J. Kosinski, and G. S. Roubin, "A multicenter randomized trial comparing a percutaneous collagen hemostasis device with conventional manual compression after diagnostic angiography and angioplasty," Journal of the American College of Cardiology, vol. 22, no. 5, pp. 1273-1279, 1993.

[12] B. V. Scheer, A. Perel, and U. J. Pfeiffer, "Clinical review: complications and risk factors of peripheral arterial catheters used for haemodynamic monitoring in anaesthesia and intensive care medicine," Critical Care, vol. 6, no. 3, pp. 198-204, 2002.

[13] R. Schlierf, U. Horst, M. Ruhl, T. Schmitz-Rode, W. Mokwa, and U. Schnakenberg, "A fast telemetric pressure and temperature sensor system for medical applications," Journal of Micromechanics and Microengineering, vol. 17, no. 7, article S06, pp. S98S102, 2007.

[14] S. Silber, A. Bjorvik, H. Muhling, and A. Rosch, "Usefulness of collagen plugging with VasoSeal after PTCA as compared to manual compression with identical sheath dwell times," Catheterization and Cardiovascular Diagnosis, vol. 43, no. 4, pp. 421427, 1998.

[15] D. R. Tavris, Y. Wang, S. Jacobs et al., "Bleeding and vascular complications at the femoral access site following percutaneous coronary intervention (PCI): an Evaluation of Hemostasis Strategies," Journal of Invasive Cardiology, vol. 24, no. 7, pp. 328-334, 2012.

[16] F. Arnaud, K. Teranishi, T. Tomori, W. Carr, and R. McCarron, "Comparison of 10 hemostatic dressings in a groin puncture model in swine," Journal of Vascular Surgery, vol. 50, no. 3, pp. 632-639, 2009. 


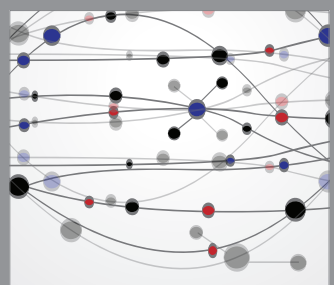

The Scientific World Journal
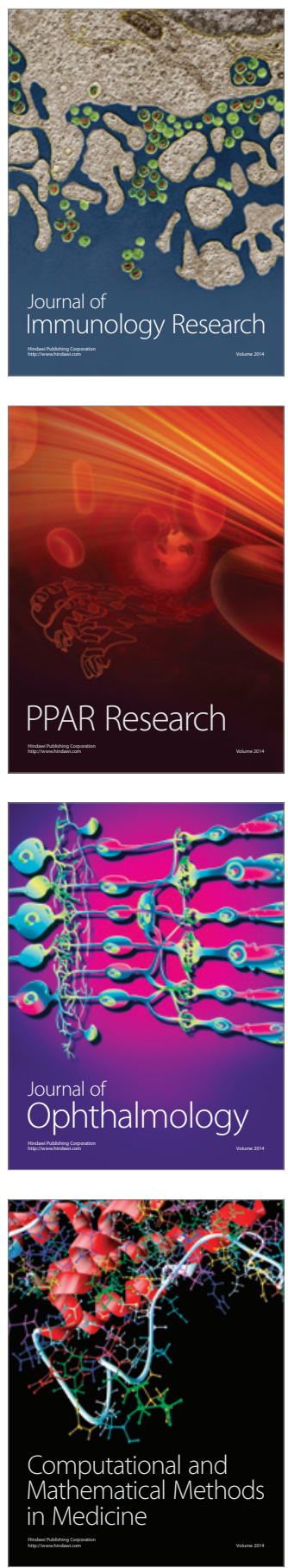

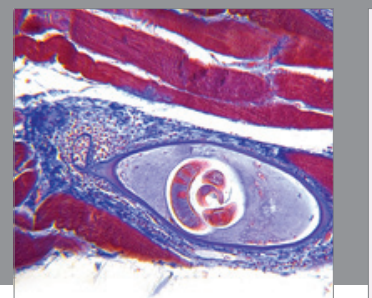

Gastroenterology

Research and Practice


\section{Hindawi}

Submit your manuscripts at

http://www.hindawi.com
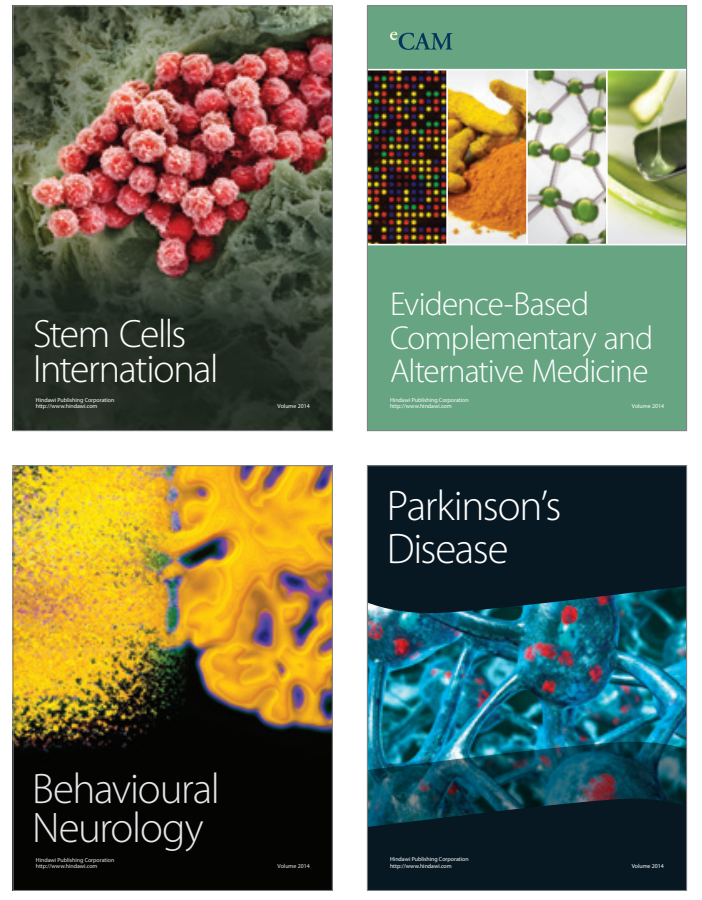
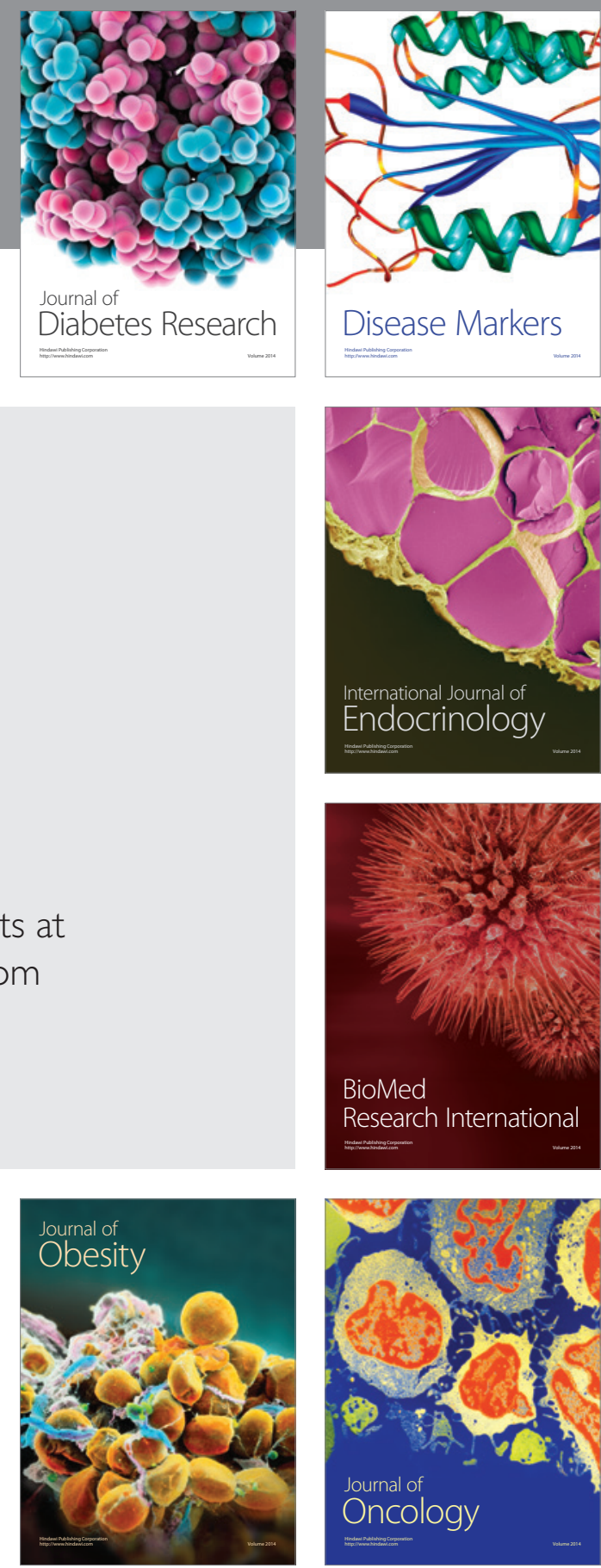

Disease Markers
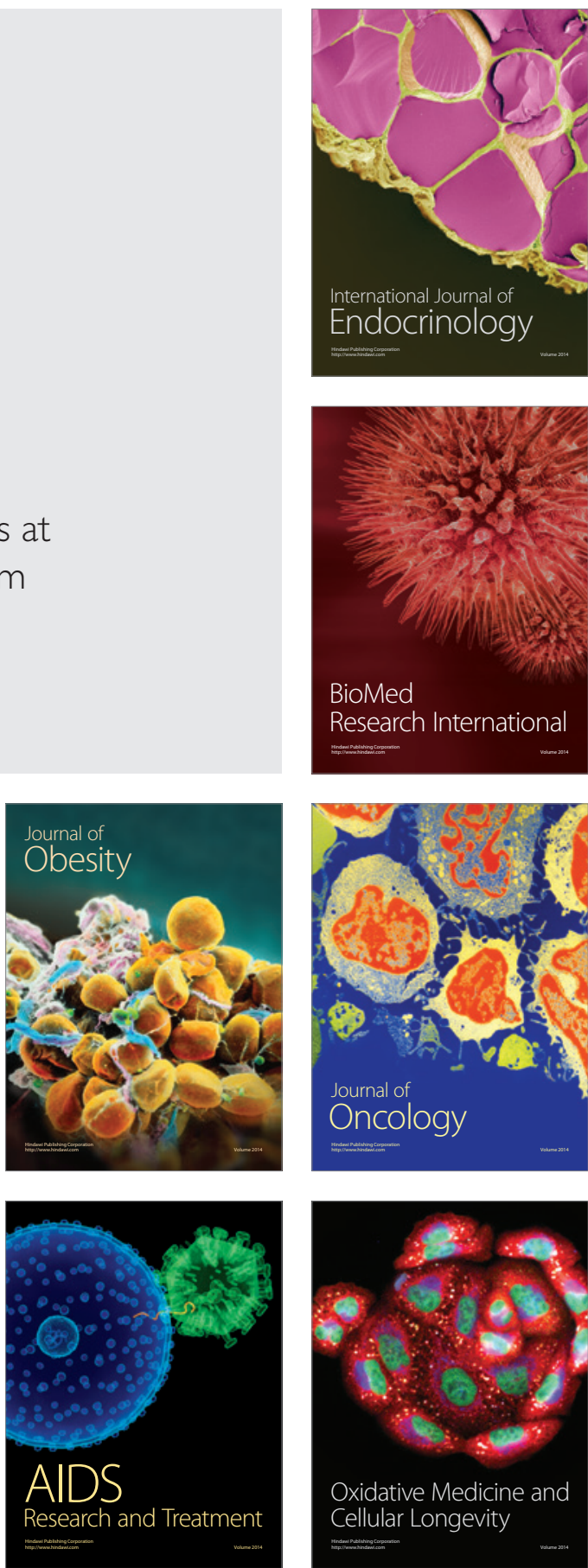